\title{
Uncertainty Quantification using Maximum Likelihood: Experimental Validation
}

\author{
José R. Fonseca* \\ School of Engineering, University of Wales Swansea, Swansea SA2 8PP, UK \\ Michael I. Friswell ${ }^{\dagger}$ \\ Department of Aerospace Engineering, University of Bristol, Bristol BS8 1TR, UK \\ John E. Mottershead ${ }^{\ddagger}$ \\ Department of Engineering, University of Liverpool, Liverpool L69 3GH, UK \\ Arthur W. Lees ${ }^{\S}$ \\ School of Engineering, University of Wales Swansea, Swansea SA2 8PP, UK \\ and \\ Sondipon Adhikari** \\ Department of Aerospace Engineering, University of Bristol, Bristol BS8 1TR, UK
}

\begin{abstract}
Parameter uncertainty is extensively used to analyze the robustness and reliability of a structure. However this analysis requires the specification of the uncertainty, and for many parameters this is not an easy quantity to estimate. This paper uses a maximum likelihood approach, combined with either a perturbation or Monte Carlo method for the forward propagation of uncertainty, to estimate the parameter mean and variance. The method is demonstrated on two experimental examples, namely cantilever and free-free beams with a moving mass.
\end{abstract}

\section{Nomenclature}

EI $\quad=$ beam stiffness

$k=$ factor multiplying beam stiffness

$L \quad=$ likelihood function

$l \quad=\log$ likelihood

$N \quad=$ number of measurement sets

$m \quad=$ number of measurements within a sample set

$\mathbf{x}=$ physical parameters

$\mathbf{y} \quad=$ response vector

$\theta=$ parameters to define the PDF of the physical parameters

$\mu, \Sigma=$ mean vector and covariance matrix

\section{Introduction}

The propagation of uncertainty in material or geometrical parameters through a finite element model to determine the uncertainty in the response has become a valuable and popular technique. Examples of the use of this approach range from robust design to reliability analysis, and computer code is available for stochastic finite

*PhD student, School of Engineering, mejmrf@swan.ac.uk.

† Sir George White Professor of Aerospace Engineering, m.i.friswell@bristol.ac.uk, AIAA Member.

‡ Alexander Elder Professor of Applied Mechanics, Department of Engineering, j.e.mottershead@liv.ac.uk.

$\S$ Professor of Mechanical Engineering, School of Engineering, a.w.lees@swansea.ac.uk.

*** Lecturer, Department of Aerospace Engineering, s.adhikari@bristol.ac.uk, AIAA Member. 
element analysis and uncertainty propagation (examples of the latter are NESSUS and ST-ORM). These techniques work extremely well and give great insight into the robustness of structures. However all of these approaches rely on knowledge of the parameter uncertainty. For many parameters, such as panel thicknesses, direct measurement may be used to get the description of the uncertainty in terms of a probably density function. Tolerances may be used to give some insight into the variance for probabilistic approaches or the bounds for possiblistic approaches. However, direct measurement of many parameters is not possible. For example, the model of many joints is uncertain, but the nature of the modeling errors means that equivalent models must be used, based on generic elements, geometric parameters, or other techniques ${ }^{1,2}$. The uncertainty in the parameters must be obtained from measurements, using inverse methods ${ }^{3}$, and this is the subject of this paper.

\section{Uncertainty Quantification using Maximum Likelihood}

The approach adopted in this paper is to parameterize the probability density function of the parameters and to estimate these parameters from multiple measured data sets. For example, if the parameters were assumed to be taken from a Gaussian distribution then the mean vector and covariance matrix is all that is required. This is essentially a form of regularization, since a smaller number of parameters are estimated for a given measured data set. The important issues are the form of the penalty function, which will be based on a maximum likelihood criterion, and the evaluation of the penalty function through perturbation and Monte Carlo approaches. Note that this method is the only one possible to deal with random fields, where the uncertainty is spatially distributed and may be simply parameterized by a correlation length (in the simplest model).

\section{A. Maximum Likelihood}

The key to the maximum likelihood approach is to parameterize the probability density functions (PDFs) of the parameters. The procedure is to determine the probability that the measurements occur given the PDF of the parameters. An optimization procedure, such as the simplex algorithm, may then be used to determine the optimum parameters describing the PDFs.

Suppose that the physical parameters, $\mathbf{x}$, follow a certain probability distribution belonging to a probability distribution family parameterized by $\theta$ (for example the mean, $\mu$, and covariance matrix, $\Sigma$ ). For a given $\theta$, the output PDF, $f(\mathbf{y} \mid \theta)$, can be approximated using one of the uncertainty propagation methods. Let the measurements be $\mathbf{y}_{1}, \mathbf{y}_{2}, \ldots, \mathbf{y}_{N}$. The measurements are assumed to be independent, therefore the measurements likelihood is

$$
L(\theta)=f\left(\mathbf{y}_{1}, \mathbf{y}_{2}, \ldots, \mathbf{y}_{N} \mid \theta\right)=\prod_{i=1}^{N} f\left(\mathbf{y}_{i} \mid \theta\right)
$$

or taking logarithms,

$$
l(\theta)=\log L(\theta)=\sum_{i=1}^{N} \log f\left(\mathbf{y}_{i} \mid \theta\right)
$$

The maximum likelihood estimator, $\hat{\theta}$, is the value of $\theta$ for which $l(\theta)$ is a maximum. A non-gradient based optimization method, such as the simplex method, can be employed to allow the use of standard uncertainty propagation methods. The drawback of this approach is its iterative nature. The uncertainty propagation methods are by themselves computationally intensive and to execute one in an iterative optimization loop would be prohibitive for most interesting applications. Ways to efficiently integrate the maximum likelihood estimation with the two most common propagation methods are discussed below. Fonseca et al. ${ }^{4}$ has more detail.

\section{B. Perturbation Method}

In the perturbation approach, the response outputs, $\mathbf{y}$, are expanded as a Taylor series in the physical parameters $\mathbf{x}$, about the parameters $\mathbf{x}_{0}$. Thus if $\mathbf{y}=\mathbf{g}(\mathbf{x})$, then 


$$
\mathbf{y}=\mathbf{g}\left(\mathbf{x}_{0}\right)+\mathbf{J}_{0}\left(\mathbf{x}-\mathbf{x}_{0}\right)+\text { higher order terms }
$$

where $\mathbf{J}_{0}$ is the sensitivity or Jacobian matrix evaluated at $\mathbf{x}_{0}$. If the physical parameters are derived from a Gaussian distribution with mean $\mu$ and covariance $\Sigma$, denoted $N(\mu, \Sigma)$, then $\mathbf{y}$ is

$$
N\left(\mu_{y}=\mathbf{g}\left(\mathbf{x}_{0}\right)+\mathbf{J}_{0}\left(\mu-\mathbf{x}_{0}\right), \Sigma_{y}=\mathbf{J}_{0}^{T} \Sigma \mathbf{J}_{0}\right) .
$$

Using the standard PDF for the Gaussian distribution produces the log likelihood function as

$$
l(\mu, \Sigma)=-\frac{1}{2}\left(N m \log 2 \pi+N \log \left|\Sigma_{y}\right|+\sum_{i=1}^{N}\left(\mathbf{y}_{i}-\mu_{y}\right)^{T} \Sigma_{y}^{-1}\left(\mathbf{y}_{i}-\mu_{y}\right)\right),
$$

where $m$ is the number of elements in the vector $\mathbf{y}$, and this is a straight-forward optimization problem for the parameter mean $\mu$ and covariance $\Sigma$.

Ideally $\mathbf{x}_{0}$ would be the mean $\mu$ but the mean is not known beforehand, so a guess must be made for its initial value. Depending how far that initial guess is from the estimated mean $\hat{\mu}$, it may be necessary to recompute $\mathbf{g}\left(\mathbf{x}_{0}\right)$ and $\mathbf{J}_{0}$ to more accurately describe the response surface near $\mu$. However, this computation does not have to be performed for every evaluation of Eq. (5). For most applications an approximate knowledge of the mean value is available reducing the need for such calculations.

\section{Monte Carlo Method}

The Monte Carlo approach samples physical parameters based on the assumed PDF. These samples are used to calculate the response of the structure and the PDF of $\mathbf{y}$ may then be approximated using a kernel density estimator. Using this standard method is very time consuming because when the parameters describing the PDF of $\mathbf{x}$ (such as the mean and variance) change then a new set of samples must be calculated and the results propagated through the model. The efficiency of the method may be improved significantly by retaining the results for the samples we have already computed. When the parameter PDF changes the contribution of these samples to the response PDF are simply re-weighted ${ }^{4}$. This approach has the further advantage that a reduced number of samples are required. If independent samples are taken at each iteration then determining convergence may be difficult because the sample statistics will change slightly from iteration to iteration.

\section{A Cantilever Beam with a Moving Mass}

The first example is a cantilever beam with a point mass placed at an uncertain position along the beam length. The properties of the maximum likelihood estimator have been investigated using simulated natural frequencies in a previous paper ${ }^{4}$. These earlier investigations included the effect of the number of samples, the number of natural frequencies used and the linearisation point for the perturbation approach. Here experimental data from the structure shown in Fig. 1 is used. The model was modified to account for the accelerometer (with a mass $m_{a}=34.1 \mathrm{~g}$ positioned at $x_{a}=20 \mathrm{~mm}$ from the beam free end) and to allow some clamping flexibility ( $K$ and $\left.K_{t}\right)$, as illustrated in Fig. 2. The beam has a length $l=60 \mathrm{~cm}$, a rectangular section of $70 \times 12 \mathrm{~mm}^{2}$ and is made of steel (Young's modulus $E=210 \mathrm{GPa}$ and mass density $\rho=7800 \mathrm{~kg} / \mathrm{m}^{3}$ ). The moving mass is $m=93.6 \mathrm{~g}$ and its position $x$ follows a Gaussian distribution $X \rightarrow N(\mu=15 \mathrm{~cm}, \sigma=5 \mathrm{~cm})$.

The clamping stiffnesses $K=91.466 \mathrm{MNm}$ and $K_{t}=109.825 \mathrm{kNrad}$ were determined by model updating of the beam without the mass ${ }^{3}$, by minimizing the relative error sum of the first three natural frequencies (Table 1). 50 samples of $x$ were generated and rounded to the nearest $\mathrm{mm}$. The mass was positioned accordingly and the measurements of the first natural frequency taken, which are shown in Fig. 3. Even after model updating there is a small bias $\Delta$ between the measured natural frequencies and the ones derived by the model. To reduce these modeling errors (which would otherwise yield an offset in the mean estimate of the mass position) a bias is introduced into the system response model. 
Table 2 shows the estimates of $\mu_{X}$ and $\sigma_{X}$ compared with their real and effective counterparts, when only the first natural frequency is used for the estimation. The estimates obtained by the Monte Carlo method are only slightly closer to the effective values than those obtained by the perturbation method. Note that the closeness of the perturbation estimates to the real values is merely a coincidence due to error cancellation, i.e. the linearisation error and the sampling error happened to have opposite signs.

\section{A Free-Free Beam with a Moving Mass}

A beam of length $1 \mathrm{~m}$ and made from bright mild steel was suspended using flexible supports to simulate freefree conditions. A small mass (magnet) was attached to the beam and its position was varied using a Gaussian distribution with mean, $\mu$, of $800 \mathrm{~mm}$ and standard deviation, $\sigma$, of $30 \mathrm{~mm}$. 50 mass positions were sampled from this distribution. The experimental setup is illustrated schematically in Fig. 4. The mass properties and dimensions for the components are given in Table 3.

For each test the centreline of the mass was aligned with the relevant position along the beam. The thinnest edge of the mass was placed in contact with the beam to minimise any stiffening effects. Impact excitation was used, and the average of three runs was taken at each mass location. The response was measured with a PCB Model 333M07 accelerometer located at $20 \mathrm{~mm}$ from the beam end, and excited by a PCB impact hammer with the impact location at $417 \mathrm{~mm}$ from the accelerometer end. The data acquisition and analysis performed using an LMS Test.Lab system with a bandwidth of $512 \mathrm{~Hz}$ and 4096 frequency lines and an exponential response window. The first three natural frequencies were estimated from the FRFs using time domain parameter extraction.

Figure 5 shows the frequency response functions up to $200 \mathrm{~Hz}$ at all mass positions. This frequency range covers the first three bending modes of the beam. A rigid body mode between 2 and $3 \mathrm{~Hz}$ is clearly visible. Notice that the natural frequency of the first mode changes very little with the mass position, whereas modes 2 and 3 change significantly. For comparison a free-free beam model was created using 50 elements. Figure 6 compares the experimental and numerical FRFs when the moving mass was at its mean position of $800 \mathrm{~mm}$ from the accelerometer end. Clearly the model is reasonably close and the discrepancies arise from small differences in natural frequency caused by small differences in the flexural rigidity, EI, that are likely to be within the measurement tolerances of the beam thickness. The other significant difference is that the numerical model was undamped.

Figure 7 shows the first two natural frequencies as a function of the position of the mass. For comparison the numerical results are also shown. The natural frequencies change smoothly with mass position and, apart from the offset due to the modeling error described above, the experimental and numerical result corresponded closely. This figure also highlights the mass positions used for the experiments, which were taken from a Gaussian distribution.

In this example two parameters will be updated, namely the mass position and the beam stiffness. In the cantilever beam example the beam stiffness in the finite element model was updated deterministically at a particular point in parameter space (i.e., for a specific mass position). The beam stiffness is represented by a factor $k$, where

$$
E I=k E I_{0} .
$$

Table 4 shows the uncertainty identification results for the perturbation and Monte Carlo approaches for the three cases: when $E I$ is assumed to be its nominal value, $k=1$; when $E I$ is unknown but deterministic, $k=\mu_{k}$; or when $E I$ is random and has a Gaussian distribution, $k \rightarrow N\left(\mu_{k}, \sigma_{k}\right)$. The percentage errors are the relative errors from the effective parameters. The standard deviation estimate from the perturbation approach is not affected by the errors in the mean of the beam stiffness between the model and measurements (in both instances $\mu_{X}$ is estimated to be 0.02336). However the estimate of the mean mass position is affected. This characteristic may be useful for applications where only the variation is sought, for example when determining the source of variability. The Monte Carlo method (which effectively models the non-linear relationship between the parameters and the response) is sensitive to modeling errors. The estimation error in the mass position mean and standard deviation reduces from $18 \%$ and $99 \%$ down to $2 \%$ and $5 \%$, respectively, when $E I$ is allowed to vary. Thus, once the model and experiment are well correlated the Monte Carlo gives excellent results for the mass position distribution.

To determine the ability of the methods to identify sources of variability (without prior knowledge concerning which parameter might vary), $k$ (and hence $E I$ ) was also taken as a random variable with a Gaussian distribution. Of course the beam used throughout the experiment remained the same, and therefore EI should not vary. The Monte 
Carlo approach performed well, estimating a negligible value for the standard deviation, $\sigma_{X}=3.255 \times 10^{-4}$. The perturbation approach performs poorly, and attributes to a significant part of the variability to $E I$.

\section{Conclusion}

This paper has given some insight into the issues involved in the quantification of parameter uncertainty. The most promising approach is to parameterize the probability density function of the parameters (for example using the mean and covariance) and to identify these parameters using the maximum likelihood method. The perturbation approach has problems when the linear approximation to the response is not very accurate, and can lead to more information giving higher parameter estimation errors. The Monte Carlo approach works well, but the algorithm must be carefully designed to ensure that the computational effort is realistic. The approaches have been tested on two experimental examples, namely a moving mass on cantilever and free-free beams.

The FRF data, and natural frequency data for the free-free beam example, may be downloaded from

$$
\text { http://www.aer.bris.ac.uk/research/uncertainty/ }
$$

\section{Acknowledgments}

The authors acknowledge the support of the Engineering and Physical Sciences Research Council (United Kingdom) through grants GR/R34936 and GR/R26818. Friswell acknowledges the support of a Royal SocietyWolfson Research Merit Award. Foncesa acknowledges the support of the Portuguese Foundation for Science and Technology through the scholarship SFRH/BD/7065/2001.

\section{References}

${ }^{1}$ Friswell, M. I., Mottershead, J. E. and Ahmadian, H., "Finite Element Model Updating using Experimental Test Data: Parameterisation and Regularisation," Transactions of the Royal Society of London, Series A: Mathematical, Physical and Engineering Sciences, special issue on Experimental Modal Analysis, Vol. 359, 2001, pp. 169-186.

${ }^{2}$ Mottershead, J. E., Friswell, M. I., Ng, G. H. T. and Brandon, J. A., "Geometric Parameters for Finite Element Model Updating of Joints and Constraints,” Mechanical Systems and Signal Processing, Vol. 10, No. 2, 1996, pp. 171-182.

${ }^{3}$ Friswell, M. I. and Mottershead, J. E., Finite Element Model Updating in Structural_Dynamics, Kluwer Academic Publishers, Dordrecht, 1995.

${ }^{4}$ Fonseca, J. R., Friswell, M. I., Mottershead, J. E. and Lees, A. W., "Uncertainty Identification by the Maximum Likelihood Method," Journal of Sound and Vibration (in press).
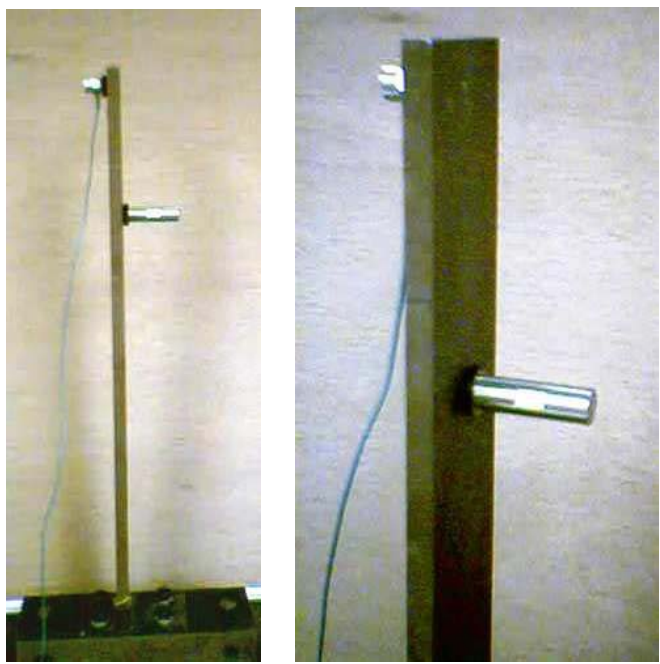

Figure 1. Experimental setup for the cantilever beam with a lumped mass at an uncertain position. 


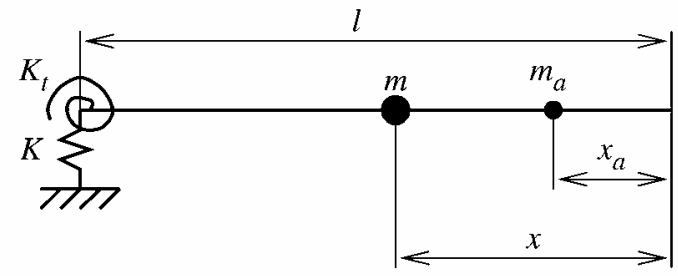

Figure 2. The model of the cantilever beam with a lumped mass at an uncertain position.

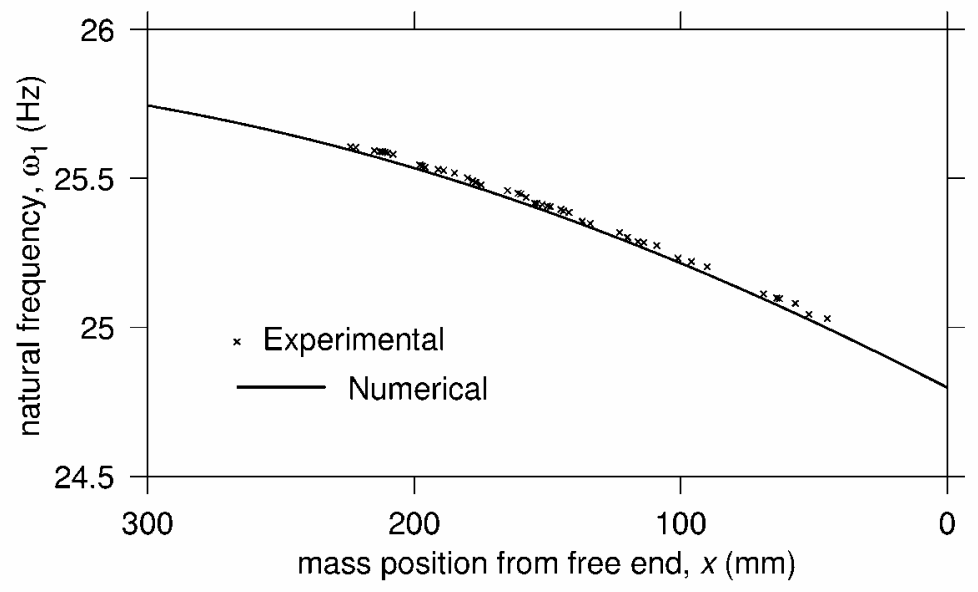

Figure 3. The first natural frequency of the cantilever beam (experimental vs. model).

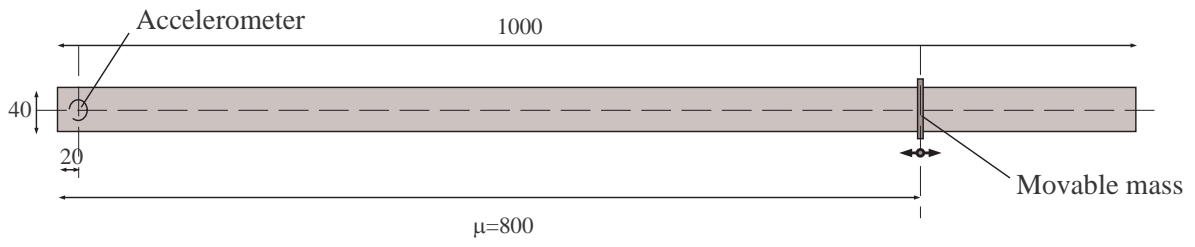

All dimensions in mm

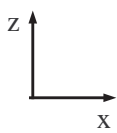

Figure 4. Experimental setup for the free-free beam. 


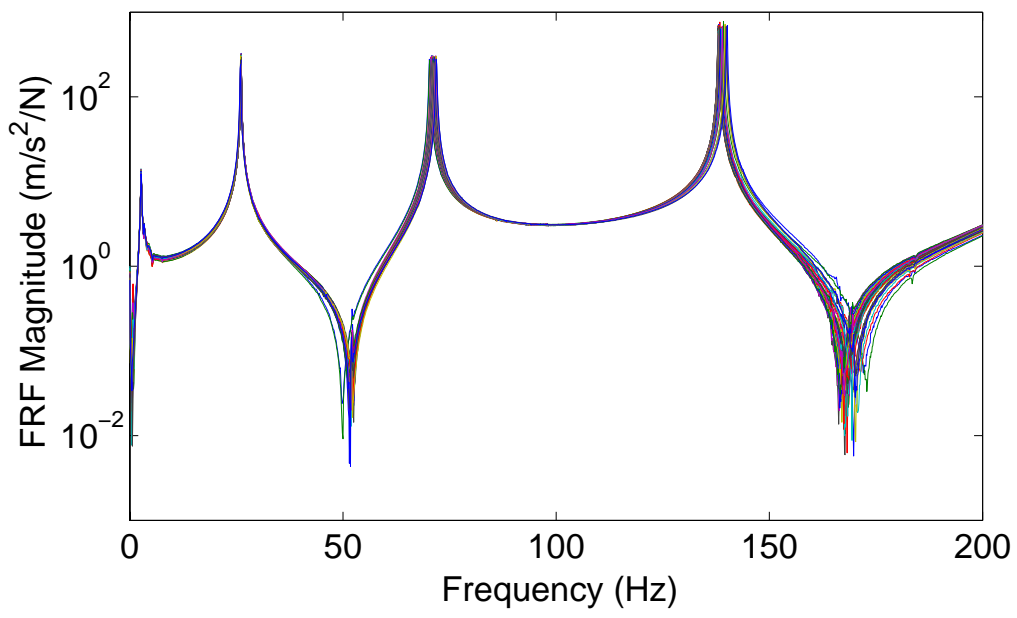

Figure 5. Experimental FRFs for all mass positions for the free-free beam example.

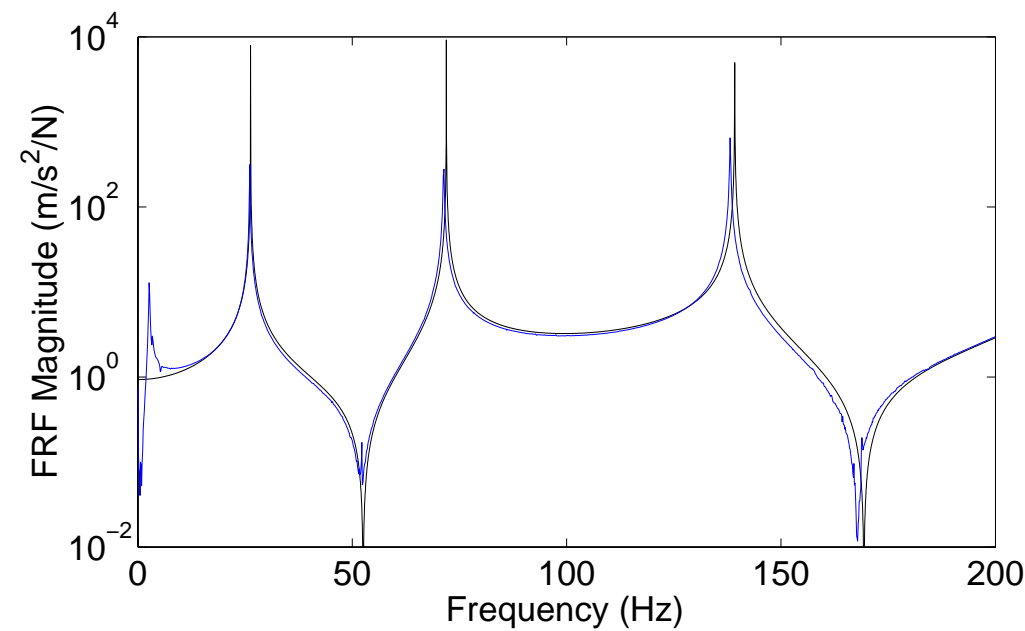

Figure 6. Comparison of experimental and numerical FRFs for the mean mass position for the free-free beam example.
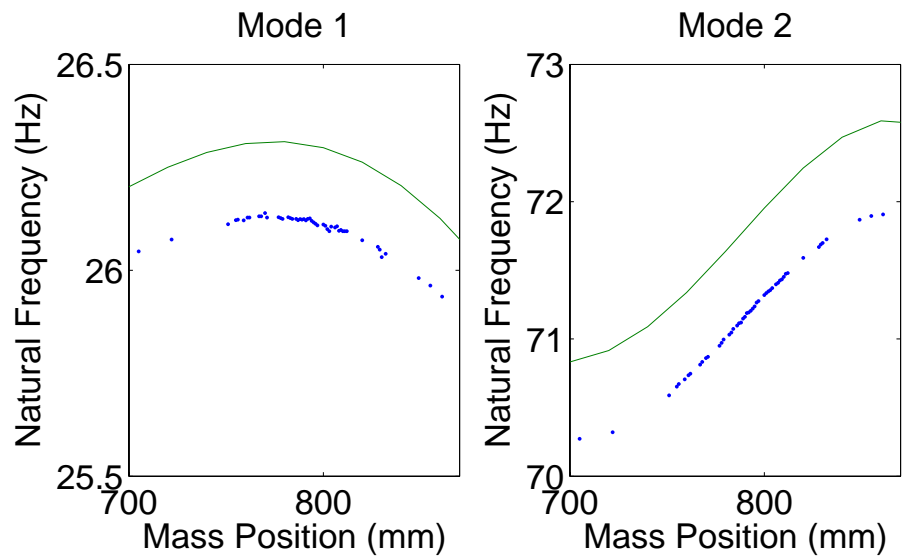

Figure 7. The variation of natural frequency with mass position for the free-free beam example (the dots denote experimental data points). 
Table 1. Model updating of the clamping stiffnesses of the cantilever beam without the mass.

\begin{tabular}{|c|c|c|c|}
\hline Mode & Measured $(\mathrm{Hz})$ & Updated $(\mathrm{Hz})$ & $\Delta(\mathrm{Hz})$ \\
\hline 1 & 25.9049 & 25.8906 & -0.0143 \\
\hline 2 & 162.9649 & 163.1180 & +0.1532 \\
\hline 3 & 456.7434 & 456.5961 & -0.1473 \\
\hline 4 & 890.0572 & 889.5894 & -0.4678 \\
\hline
\end{tabular}

Table 2. Estimation results for the moving mass on a cantilever beam.

\begin{tabular}{|l|c|c|}
\hline & $\mu_{X}(\mathrm{~mm})$ & $\sigma_{X}(\mathrm{~mm})$ \\
\hline Real & 150.0 & 50.0 \\
\hline Effective & 151.5 & 49.5 \\
\hline Perturbation estimate & 149.5 & 50.4 \\
\hline Monte Carlo estimate & 153.4 & 48.5 \\
\hline
\end{tabular}

Table 3. Component masses and dimensions for the free-free beam example.

\begin{tabular}{|l|c|c|c|c|}
\hline Component & Mass $(\mathrm{g})$ & $x(\mathrm{~mm})$ & $y(\mathrm{~mm})$ & $z(\mathrm{~mm})$ \\
\hline Beam & 1561 & 1000 & 40 & 5 \\
\hline Mass (Magnet) & 47 & 5 & 50 & 25 \\
\hline Accelerometer & 6 & & & \\
\hline
\end{tabular}

Table 4. Estimation results for the moving mass on a free-free beam.

\begin{tabular}{|l|c|c|c|c|}
\hline & \multicolumn{1}{|c|}{$\mu_{X}(\mathrm{~m})$} & \multicolumn{1}{|c|}{$\sigma_{X}(\mathrm{~m})$} & $\mu_{k}$ & $\sigma_{k}$ \\
\hline Real & 0.8 & 0.03 & $?$ & 0 \\
\hline Effective & 0.7921 & 0.02993 & $?$ & 0 \\
\hline Perturbation & $0.7016(-11.42 \%)$ & $0.02336(-21.95 \%)$ & - & - \\
\hline Monte Carlo & $0.9398(18.72 \%)$ & $0.00003(-98.67 \%)$ & - & - \\
\hline Perturbation & $0.8717(10.04 \%)$ & $0.02336(-21.95 \%)$ & 0.9487 & - \\
\hline Monte Carlo & $0.7929(0.11 \%)$ & $0.02858(-4.52 \%)$ & 1.0249 & - \\
\hline Perturbation & $0.7724(-2.48 \%)$ & $0.03469(15.88 \%)$ & 0.9786 & $1.022 \times 10^{-2}$ \\
\hline Monte Carlo & $0.7938(0.21 \%)$ & $0.02905(-2.93 \%)$ & 0.9710 & $3.255 \times 10^{-4}$ \\
\hline
\end{tabular}

